\title{
SOME REMARKS ON A SHAPE OPTIMIZATION PROBLEM
}

\author{
FRANCESCO DELLA PIETRA
}

Abstract. Given $\Omega$ bounded open set of $\mathbb{R}^{n}$ and $\alpha \in \mathbb{R}$, let us consider

$$
\mu(\Omega, \alpha)=\min _{\substack{v \in W_{0}^{1,2}(\Omega) \\ v \neq 0}} \frac{\int_{\Omega}|\nabla v|^{2} d x+\alpha\left|\int_{\Omega}\right| v|v d x|}{\int_{\Omega}|v|^{2} d x} .
$$

We study some properties of $\mu(\Omega, \alpha)$ and of its minimizers, and, depending on $\alpha$, we determine the set $\Omega_{\alpha}$ among those of fixed measure such that $\mu\left(\Omega_{\alpha}, \alpha\right)$ is the smallest possible.

\section{Statement of the problem and main Result}

Let $\Omega$ be a bounded open set of $\mathbb{R}^{n}, n \geq 2$, and consider the following minimum problem

$$
\mu(\Omega, \alpha)=\min _{\substack{v \in W_{0}^{1,2}(\Omega) \\ v \neq 0}} \mathcal{Q}(v, \alpha)
$$

where $\alpha$ is a fixed real number and

$$
\mathcal{Q}(v, \alpha)=\frac{\int_{\Omega}|\nabla v|^{2} d x+\alpha\left|\int_{\Omega}\right| v|v d x|}{\int_{\Omega}|v|^{2} d x} .
$$

The objective of this paper is to study some properties of $\mu(\Omega, \alpha)$ and of its minimizers. Moreover, we aim to determine and to characterize the sets $\tilde{\Omega}$ among those of fixed measure such that $\mu(\tilde{\Omega}, \alpha)$ is the smallest possible. As we will show, the shape of $\tilde{\Omega}$ depends on $\alpha$. More precisely if we denote, as usual, by $\omega_{n}$ the measure of the unit ball in $\mathbb{R}^{n}$, and by $j_{n / 2-1,1}$ the first zero of the Bessel function of first kind of order $n / 2-1$, the main result of the paper is the following.

Theorem 1.1. Let $n \geq 2$. There exists a positive number

$$
\alpha_{c}=j_{n / 2-1,1}^{2} \omega_{n}^{\frac{2}{n}}\left[2^{\frac{2}{n}}-1\right]
$$

such that, for every bounded open set $\Omega \subset \mathbb{R}^{n}$ and for every $\alpha \in \mathbb{R}$, it holds

$$
\mu(\Omega, \alpha) \geq \begin{cases}\mu\left(\Omega^{\#}, \alpha\right) & \text { if } \alpha|\Omega|^{\frac{2}{n}} \leq \alpha_{c}, \\ \frac{2^{2 / n} \omega_{n}^{2 / n} j_{n / 2-1,1}^{2}}{|\Omega|^{2 / n}} & \text { if } \alpha|\Omega|^{\frac{2}{n}} \geq \alpha_{c},\end{cases}
$$

where $\Omega^{\#}$ is the ball centered at the origin with measure $\left|\Omega^{\#}\right|=|\Omega|$. If the equality sign holds when $\alpha|\Omega|^{\frac{2}{n}}<\alpha_{c}$, then $\Omega$ is a ball. If the equality sign holds when $\alpha|\Omega|^{\frac{2}{n}}>\alpha_{c}$, then $\Omega$ is the union of two disjoint balls of equal measure. If $\alpha|\Omega|^{\frac{2}{n}}=\alpha_{c}$ and the equality sign holds, $\Omega$ is a ball or the union of two disjoint balls of equal measure.

Date: August 19, 2018.

2010 Mathematics Subject Classification. 35P15, 49R50.

Key words and phrases. Eigenvalues; Shape optimization; Symmetrization. 
On the other hand, the above result provides the best constant $\mu(\tilde{\Omega}, \alpha)$ in the corresponding Sobolev-Poincaré inequality:

$$
\mu(\tilde{\Omega}, \alpha) \int_{\Omega}|v|^{2} d x \leq \int_{\Omega}|\nabla v|^{2} d x+\alpha\left|\int_{\Omega}\right| v|v d x|, \quad v \in W_{0}^{1,2}(\Omega),
$$

among all the open bounded sets $\Omega$ with fixed measure.

Let us observe that when $\alpha=0$, the above inequality reduces to the classical Poincaré inequality. Moreover, $\mathcal{Q}(\Omega, 0)$ is the Rayleigh quotient associated to the Dirichlet Laplacian eigenvalue problem, and $\mu(\Omega, 0)$ corresponds to its first eigenvalue in $\Omega$. Then, it is well known the Faber-Krahn inequality:

$$
\mu(\Omega, 0) \geq \mu\left(\Omega^{\#}, 0\right) .
$$

Moreover if the equality replaces the inequality, then $\Omega$ is a ball.

The problem of finding the optimal shape of set-dependent functionals is largely studied in many settings. Several results can be found for example in [14], related to eigenvalue problems, or in [16]. Recent results are contained for example in [1,3,5,13, 17. Moreover, we recall that in [2] a result analogous to Theorem 1.1 is given for the functional

$$
\tilde{\lambda}(\Omega, \alpha)=\min _{\substack{v \in W_{0}^{1,2}(\Omega) \\ v \neq 0}} \frac{\int_{\Omega}|\nabla v|^{2} d x+\alpha\left(\int_{\Omega} v d x\right)^{2}}{\int_{\Omega}|v|^{2} d x},
$$

which is related to a nonlocal eigenvalue problem. It has been proved that there exists a threshold positive value $\tilde{\alpha}$ such that if $\alpha<\tilde{\alpha}$, the minimum of $\tilde{\lambda}(\Omega, \alpha)$ among the sets with fixed measure is attained at one ball, while for $\alpha$ greater than $\tilde{\alpha}$, such minimum is given at two balls of equal measure.

The paper is organized as follows. In Section 2, we recall some basic results on Schwarz symmetrization and on the Dirichlet Laplacian. Moreover, depending on $\alpha$, we give some properties of $\mu(\Omega, \alpha)$ and its minimizers. Finally, in Section 3 we give the proof of the main result.

\section{Notation AND PRELIMINARY RESUlts}

2.1. Schwarz symmetrization. Let $\Omega$ be a bounded open set of $\mathbb{R}^{n}$ and $u: \Omega \rightarrow \mathbb{R}$ be a measurable function, and denote by $\Omega^{\#}$ the ball centered at the origin with the same Lebesgue measure of $\Omega$. The Schwarz rearrangement of $u$ is the spherically symmetric decreasing function

$$
u^{\#}: \Omega^{\#} \rightarrow[0,+\infty[
$$

whose level sets are balls have the same measure of the level sets of $|u|$, that is

$$
\left|\left\{u^{\#}>t\right\}\right|=|\{|u|>t\}|, \quad t \geq 0 .
$$

The Schwarz symmetrization enjoys the following properties.

a) By definition, $u^{\#}$ preserves the $L^{p}$-norm of $u$ :

$$
\|u\|_{L^{p}(\Omega)}=\left\|u^{\#}\right\|_{L^{p}\left(\Omega^{\#}\right)}, \quad 1 \leq p \leq+\infty .
$$

b) The Pólya-Szego inequality holds: if $u \in W_{0}^{1,2}(\Omega)$ is a nonnegative function, then

$$
\int_{\Omega}|\nabla u|^{2} d x \geq \int_{\Omega^{\#}}\left|\nabla u^{\#}\right|^{2} d x .
$$

Moreover, if the above inequality becomes an equality, and

$$
\left|\left\{\left|\nabla u^{\#}\right|=0\right\} \cap\left(u^{\#}\right)^{-1}(0, \operatorname{ess} \sup u)\right|=0,
$$

then, up to translations, $\Omega=\Omega^{\#}$ and $u=u^{\#}$ almost everywhere (see [4]). 
For an exhaustive treatment on rearrangements and symmetrization, we refer the reader, for example, to [15].

2.2. Some basic facts for the Dirichlet Laplacian. Given $G \subset \mathbb{R}^{n}$ bounded open set, throughout the paper we will denote by $\lambda_{\Delta}(G)$ the first Dirichlet-Laplace eigenvalue relative to $G$ :

$$
\lambda_{\Delta}(G)=\min _{v \in W_{0}^{1,2}(G) \backslash\{0\}} \frac{\int_{G}|\nabla v|^{2} d x}{\int_{G}|v|^{2} d x},
$$

and by $\lambda_{T}(G)$ the minimum of the constrained problem

$$
\lambda_{T}(G)=\min _{\substack{v \in W_{0}^{1,2}(G) \backslash\{0\} \\ \int_{G}|v| v d x=0}} \frac{\int_{G}|\nabla v|^{2} d x}{\int_{G}|v|^{2} d x} .
$$

As regards (2.3), we recall some basic properties:

(1) The Faber-Krahn inequality: for any bounded open set in $\Omega \subset \mathbb{R}^{n}$, it holds that

$$
\lambda(\Omega) \geq \lambda\left(\Omega^{\#}\right)=\frac{\omega_{n}^{2 / n}}{|\Omega|^{2 / n}} j_{n / 2-1,1}^{2},
$$

where $j_{n / 2-1,1}$ denotes, as usual, the first zero of the Bessel function of first kind of order $n / 2-1$. If equality sign holds, then $\Omega$ is a ball.

(2) If $\Omega=B_{1} \cup B_{2}$ is the union of two disjoint balls $B_{1}, B_{2}$ with different radii $R_{1}>R_{2}>0$, then

$$
\lambda_{\Delta}(\Omega)=\lambda_{\Delta}\left(B_{1}\right)=\frac{j_{n / 2-1,1}^{2}}{R_{1}^{2}} .
$$

Hence it is simple, any associated eigenfuction does not change sign in the largest ball $B_{1}$, and it is identically zero in $B_{2}$.

(3) If $\Omega=B_{1} \cup B_{2}$ is the union of two disjoint balls $B_{1}, B_{2}$ with equal radii $0<R_{1}=R_{2}$, the first eigenvalue is not simple, and there exists an eigenfunction $u$ positive in $B_{1}$, negative in $B_{2}$ and such that $\int_{B_{1} \cup B_{2}}|u| u d x=0$. In particular, this eigenfunction coincides with the positive first eigenfunction of $\lambda_{\Delta}\left(B_{1}\right)$, and to its opposite (up to a translation) in $B_{2}$.

2.3. Some properties of $\mu(\Omega, \alpha)$. In what follows, for a given function $u: \Omega \rightarrow \mathbb{R}, u_{+}=$ $\max \{u, 0\}$ and $u_{-}=\max \{-u, 0\}$ will be its positive and negative part, and

$$
\Omega_{+}=\left\{u_{+}>0\right\}, \quad \Omega_{-}=\left\{u_{-}>0\right\} .
$$

Proposition 2.1. The following properties for $\mu(\Omega, \alpha)$ hold.

(a) The minimum $\mu(\Omega, \alpha)$ is 1-Lipschitz continuous and it is non-decreasing with respect to $\alpha \in \mathbb{R}$.

(b) For $\alpha<0$,

$$
\mu(\Omega, \alpha)=\lambda_{\Delta}(\Omega)+\alpha
$$

(c) For $\alpha \geq 0$,

$$
\lambda_{\Delta}(\Omega) \leq \mu(\Omega, \alpha) \leq \min \left\{\lambda_{T}(\Omega), \lambda_{\Delta}(\Omega)+\alpha\right\} .
$$

(d) As $\alpha \rightarrow+\infty$, we have that

$$
\lim _{\alpha \rightarrow+\infty} \mu(\Omega, \alpha)=\lambda_{T}(\Omega) .
$$


Proof. (a) For any $\varepsilon>0$,

$$
\mathcal{Q}(v, \alpha) \leq \mathcal{Q}(v, \alpha+\varepsilon) \leq \mathcal{Q}(v, \alpha)+\varepsilon .
$$

Taking the minimum over $W_{0}^{1,2}(\Omega) \backslash\{0\}$, we have

$$
0 \leq \mu(\Omega, \alpha+\varepsilon)-\mu(\Omega, \alpha) \leq \varepsilon
$$

and the proof of (a) is concluded.

(b) Being $\alpha<0$, we have that $\mathcal{Q}(v, \alpha) \geq \mathcal{Q}(|v|, \alpha)=\mathcal{Q}(v, 0)+\alpha \geq \lambda_{\Delta}(\Omega)+\alpha$, for any $v \in W_{0}^{1,2}(\Omega)$. On the other hand, if $u \in W_{0}^{1,2}(\Omega)$ is a nonnegative minimizer for (2.3), $\mathcal{Q}(u, \alpha)=\lambda_{\Delta}(\Omega)+\alpha$, and then necessarily $\lambda_{\Delta}(\Omega)+\alpha=\mu(\Omega, \alpha)$.

(c) It follows immediately from the definitions of $\mu, \lambda_{\Delta}$ and $\lambda_{T}$.

(d) Let $0 \leq \alpha_{k}, k \in \mathbb{N}$, be a positively divergent sequence. For any $k$, consider a minimizer $u_{k} \in W_{0}^{1,2}(\Omega)$ of (1.1) such that $\left\|u_{k}\right\|_{2}=1$. We have that

$$
\mu\left(\Omega, \alpha_{k}\right)=\int_{\Omega}\left|\nabla u_{k}\right|^{2} d x+\alpha_{k}\left|\int_{\Omega}\right| u_{k}\left|u_{k} d x\right| \leq \lambda_{T}(\Omega) .
$$

Then $u_{k}$ converges (up to a subsequence) to a function $U \in W_{0}^{1,2}(\Omega)$ strongly in $L^{2}(\Omega)$ and weakly in $W_{0}^{1,2}(\Omega)$. Moreover, $\|U\|_{L^{2}(\Omega)}=1$ and

$$
\left|\int_{\Omega}\right| u_{k}\left|u_{k}\right| \leq \frac{\lambda_{T}(\Omega)}{\alpha_{k}} \rightarrow 0 \quad \text { as } k \rightarrow+\infty
$$

which gives that $\int_{\Omega}|U| U d x=0$. On the other hand, the weak convergence in $W_{0}^{1,2}(\Omega)$ implies that

$$
\int_{\Omega}|\nabla U|^{2} d x \leq \liminf \int_{\Omega}\left|\nabla u_{k}\right|^{2} d x
$$

Finally, by definition of $\lambda_{T}(\Omega)$, and (2.5) we have

$$
\begin{aligned}
& \lambda_{T}(\Omega) \leq \int_{\Omega}|\nabla U|^{2} d x \leq \\
& \quad \leq \liminf _{k \rightarrow+\infty}\left(\int_{\Omega}\left|\nabla u_{k}\right|^{2} d x+\alpha_{k}\left|\int_{\Omega}\right| u_{k}\left|u_{k} d x\right|\right)=\lim _{k \rightarrow+\infty} \mu\left(\Omega, \alpha_{k}\right) \leq \lambda_{T}(\Omega),
\end{aligned}
$$

and the proof is completed.

Remark 2.1. Let us observe that from the above proposition, (b) gives that $\mu(\Omega, \cdot)$ is unbounded from below. Moreover, $\mu(\Omega, \alpha)=0$ corresponds to $-\alpha=\lambda(\Omega)$.

Remark 2.2. Among the properties of $\mu(\Omega, \alpha)$, we observe also that it does not have the same behavior of the usual Dirichlet Laplacian with respect to the rescaling of the domain, being also the term $\alpha$ affected of the rescaling. Indeed, while $\lambda_{\Delta}(t \Omega)=t^{-2} \lambda(\Omega)$, it holds that $\mu(t \Omega ; \alpha)=t^{-2} \mu\left(\Omega ; t^{2} \alpha\right)$.

In the proposition below, we describe some features of $\mu(\Omega, \alpha)$ by computing the associated Euler equation. Without loss of generality we may assume that a minimizer $u$ satisfies $\int_{\Omega}|u| u d x \geq 0$.

Lemma 2.1. Let $\alpha \geq 0$, and $u \in W_{0}^{1,2}(\Omega)$ be a minimizer for (1.1). Then $u_{+} \in W_{0}^{1,2}\left(\Omega_{+}\right)$ and $u_{-} \in W_{0}^{1,2}\left(\Omega_{-}\right)$are first eigenfunctions of the Dirichlet Laplacian relative to $\Omega_{+}$and $\Omega_{-}$ respectively. Moreover:

(1) suppose that $\int_{\Omega}|u| u d x>0$.

(a) If $u_{-} \equiv 0$ in $\Omega$, then

$$
\mu(\Omega, \alpha)=\lambda_{\Delta}\left(\Omega_{+}\right)+\alpha .
$$


(b) If $u_{-} \not \equiv 0$ in $\Omega$, then

$$
\mu(\Omega, \alpha)=\lambda_{\Delta}\left(\Omega_{+}\right)+\alpha=\frac{\lambda_{\Delta}\left(\Omega_{+}\right)+\lambda_{\Delta}\left(\Omega_{-}\right)}{2},
$$

and then the parameter $\alpha$ corresponds to

$$
\alpha=\frac{\lambda_{\Delta}\left(\Omega_{-}\right)-\lambda_{\Delta}\left(\Omega_{+}\right)}{2} .
$$

In both cases (a) and (b),

$$
\lambda_{\Delta}\left(\Omega_{+}\right)=\lambda_{\Delta}(\Omega),
$$

and

$$
\mu(\Omega, \alpha)=\lambda_{\Delta}(\Omega)+\alpha=\lambda_{\Delta}\left(\Omega_{+}\right)+\alpha .
$$

(2) Suppose that $\int_{\Omega}|u| u d x=0$. Then

$$
\mu(\Omega, \alpha)=\lambda_{T}(\Omega)=\frac{\lambda_{\Delta}\left(\Omega_{+}\right)+\lambda_{\Delta}\left(\Omega_{-}\right)}{2} .
$$

More precisely, if there exists $\bar{\alpha}$ such that a minimizer $\bar{u}$ of $\mu(\Omega, \bar{\alpha})$ satisfies $\int_{\Omega}|\bar{u}| \bar{u} d x=0$, then for any $\alpha>\bar{\alpha}, \bar{u}$ is a minimizer for $\mu(\Omega, \alpha)$, the equality in (2.11) holds, and $\bar{u}$ is a minimizer also for $\lambda_{T}(\Omega)$.

Proof. For sake of simplicity, here we write $\mu=\mu(\Omega, \alpha)$, and distinguish two cases.

Case 1: $\int_{\Omega}|u| u d x>0$. We have that $u$ solves

$$
\begin{cases}-\Delta u=\mu u-\alpha|u| & \text { in } \Omega, \\ u=0 & \text { on } \partial \Omega .\end{cases}
$$

If $u \geq 0$ in $\Omega$, then $u_{+}$satisfies

$$
\begin{cases}-\Delta u_{+}=(\mu-\alpha) u_{+} & \text {in } \Omega_{+} \\ u_{+}=0 & \text { on } \partial \Omega_{+} .\end{cases}
$$

The positivity of the eigenfunction $u_{+}$in $\Omega_{+}$guarantees that $\mu-\alpha$ coincides with the first eigenvalue $\lambda_{+}(\Omega)$ on $\Omega_{+}$, and then (2.6) holds. Moreover, (2.9) follows from the inequalities

$$
\lambda_{\Delta}\left(\Omega_{+}\right)+\alpha \leq \lambda_{\Delta}(\Omega)+\alpha \leq \lambda_{\Delta}\left(\Omega_{+}\right)+\alpha,
$$

obtained by substituting (2.6) in (2.5), and recalling the monotonicity of the Dirichlet-Laplace eigenvalues with respect to the inclusion of sets.

If $u$ changes sign in $\Omega$, then $u_{+}$and $u_{-}$satisfy

$$
\left\{\begin{array} { l l } 
{ - \Delta u _ { + } = ( \mu - \alpha ) u _ { + } } & { \text { in } \Omega _ { + } , } \\
{ u = 0 } & { \text { on } \partial \Omega _ { + } , }
\end{array} \text { and } \left\{\begin{array}{ll}
-\Delta u_{-}=(\mu+\alpha) u_{-} & \text {in } \Omega_{-}, \\
u_{-}=0 & \text { on } \partial \Omega_{-} .
\end{array}\right.\right.
$$

Hence

$$
\lambda_{\Delta}\left(\Omega_{+}\right)=\mu-\alpha, \quad \lambda_{\Delta}\left(\Omega_{-}\right)=\mu+\alpha,
$$

that give (2.7) and (2.8). Similarly as before, substituting (2.7) and (2.8) in (2.5) and using the monotonicity of $\lambda_{\Delta}(\cdot)$, the equality (2.9) holds. By (2.6), (2.7) and (2.9) we get also (2.10).

Case 2: $\int_{\Omega}|u| u d x=0$. First, we observe that in this case

$$
\mu(\Omega, \alpha)=\lambda_{T}(\Omega) .
$$

Indeed, by definition of $\mu$ and $\lambda_{T}$, and being $u$ an admissible function for (2.4), we have

$$
\lambda_{T}(\Omega) \geq \mu(\Omega, \alpha)=\mathcal{Q}(u, \alpha) \geq \lambda_{T}(\Omega) .
$$


Computing the Euler equation with the constraint $\int_{\Omega}|u| u d x=0$, the functions $u_{+} \in W_{0}^{1,2}\left(\Omega_{+}\right)$ and $u_{-} \in W_{0}^{1,2}\left(\Omega_{-}\right)$satisfy

$$
\left\{\begin{array} { l l } 
{ - \Delta u _ { + } = \lambda _ { + } u _ { + } } & { \text { in } \Omega _ { + } , } \\
{ u _ { + } = 0 } & { \text { on } \partial \Omega _ { + } , }
\end{array} \text { and } \left\{\begin{array}{ll}
-\Delta u_{-}=\lambda_{-} u_{-} & \text {in } \Omega_{-}, \\
u_{-}=0 & \text { on } \partial \Omega_{-},
\end{array}\right.\right.
$$

for some positive values $\lambda_{+}$and $\lambda_{-}$(see also [17]). Moreover, being $u_{+}$and $u_{-}$positive functions in $\Omega_{+}$and $\Omega_{-}$respectively, it follows that

$$
\lambda_{+}=\lambda_{\Delta}\left(\Omega_{+}\right), \quad \lambda_{-}=\lambda_{\Delta}\left(\Omega_{-}\right) .
$$

Hence, in this case we have that $\int_{\Omega_{+}} u_{+}^{2} d x=\int_{\Omega_{-}} u_{-}^{2} d x$, and from the minimality of $u$ and using (2.12) it follows that

$$
\mu(\Omega, \alpha)=\mathcal{Q}(u, \alpha)=\frac{\int_{\Omega_{+}}\left|\nabla u_{+}\right|^{2} d x+\int_{\Omega_{-}}\left|\nabla u_{-}\right|^{2} d x}{\int_{\Omega_{+}} u_{+}^{2} d x+\int_{\Omega_{-}} u_{-}^{2} d x}=\frac{\lambda_{\Delta}\left(\Omega_{+}\right)+\lambda_{\Delta}\left(\Omega_{-}\right)}{2},
$$

and (2.11) is proved. The proof of $(2)$ is completed by recalling that the function $\mu(\Omega, \cdot)$ is nondecreasing and bounded from above by $\lambda_{T}(\Omega)$.

Using the above lemma, the minimum $\mu(\Omega, \alpha)$ can be characterized as follows.

Proposition 2.2. If $\Omega$ is a bounded open set of $\mathbb{R}^{n}$, then

$$
\mu(\Omega, \alpha)=\min \left\{\lambda_{\Delta}(\Omega)+\alpha, \lambda_{T}(\Omega)\right\}= \begin{cases}\lambda_{\Delta}(\Omega)+\alpha, & \text { if } \alpha \leq \lambda_{T}(\Omega)-\lambda_{\Delta}(\Omega), \\ \lambda_{T}(\Omega), & \text { if } \alpha \geq \lambda_{T}(\Omega)-\lambda_{\Delta}(\Omega) .\end{cases}
$$

Proof. Let $\alpha \geq 0$ be fixed. We have to show that $\mu(\Omega, \alpha)=\min \left\{\lambda_{\Delta}(\Omega)+\alpha, \lambda_{T}(\Omega)\right\}$.

Clearly if $\lambda_{\Delta}(\Omega)+\alpha<\lambda_{T}(\Omega)$, a minimizer $u$ of $\mu(\Omega, \alpha)$ cannot verify $\int_{\Omega}|u| u d x=0$. Otherwise, by (2.11), and choosing a nonnegative first eigenfunction $u_{1}$ of $-\Delta$ in $\Omega$, we have

$$
Q(u, \alpha)=\mu(\Omega, \alpha)=\lambda_{T}(\Omega)>\lambda_{\Delta}(\Omega)+\alpha=\mathcal{Q}\left(u_{1}, \alpha\right),
$$

contradicting the minimality of $u$. Hence $\int_{\Omega}|u| u d x>0$, and by (2.10), $\mu(\Omega, \alpha)=\lambda_{\Delta}(\Omega)+\alpha$.

Analogously, if $\lambda_{\Delta}(\Omega)+\alpha>\lambda_{T}(\Omega)$, a minimizer $u$ necessarily satisfies $\int_{\Omega}|u| u d x=0$, and $\mu(\Omega, \alpha)=\lambda_{T}(\Omega)$.

Remark 2.3. We explicitly observe that if $\Omega$ is connected, a minimizer $u$ of $\mu(\Omega, \alpha)$ either is positive in $\Omega$ or $\int_{\Omega}|u| u d x=0$.

Assuming now that $\Omega$ is the union of two disjoint balls (possibly one ball), we have the following.

Corollary 2.1. If $\Omega=B_{1}$, with radius $R_{1}>0$, then

$$
\mu\left(B_{1} ; \alpha\right)= \begin{cases}\frac{j_{n / 2-1,1}^{2}}{R_{1}^{2}}+\alpha, & \text { if } \alpha \leq \lambda_{T}\left(B_{1}\right)-\frac{j_{n / 2-1,1}^{2}}{R_{1}^{2}}, \\ \lambda_{T}\left(B_{1}\right) & \text { otherwise. }\end{cases}
$$

If $\Omega=B_{1} \cup B_{2}$, where $B_{1}$ and $B_{2}$ are disjoint balls with radii $R_{1}, R_{2}$ such that $R_{1} \geq R_{2}>0$, then

$$
\mu\left(B_{1} \cup B_{2}, \alpha\right)= \begin{cases}\frac{j_{n / 2-1,1}^{2}}{R_{1}^{2}}+\alpha, & \text { if } \alpha \leq \lambda_{T}\left(B_{1} \cup B_{2}\right)-\frac{j_{n / 2-1,1}^{2}}{R_{1}^{2}}, \\ \lambda_{T}\left(B_{1} \cup B_{2}\right) & \text { otherwise. }\end{cases}
$$


In particular, if $R_{1}=R_{2}$, for any $\alpha \geq 0$

$$
\mu\left(B_{1} \cup B_{2} ; \alpha\right)=\lambda_{T}\left(B_{1} \cup B_{2}\right)=\frac{2^{\frac{2}{n}} \omega_{n}^{\frac{2}{n}} j_{\frac{n}{2}}^{2}-1,1}{|\Omega|^{\frac{2}{n}}},
$$

where the value in the right-hand side is $\lambda_{\Delta}\left(B_{1} \cup B_{2}\right)$, and any minimizer of $\mu\left(B_{1} \cup B_{2}, \alpha\right)$ is a minimizer of $\lambda_{T}\left(B_{1} \cup B_{2}\right)$.

Proof. The proof of (2.13) and (2.14) follows from Proposition 2.2 by writing explicitly $\lambda_{\Delta}$ in the case of one ball or two disjoint balls. Then, we have only to show last equality in (2.15). Observe first that

$$
\lambda_{T}\left(B_{1} \cup B_{2}\right) \geq \lambda_{\Delta}\left(B_{1} \cup B_{2}\right) .
$$

On the other hand, being $B_{1}$ and $B_{2}$ disjoint balls with equal measure, there exists an eigenfunction $V$ of the Dirichlet Laplacian relative to $B_{1} \cup B_{2}$ such that $\int_{B_{1} \cup B_{2}}|V| V d x=0$. More precisely, this eigenfunction corresponds to a first positive Dirichlet Laplacian eigenfunction on $B_{1}$, and to its opposite (up to a translation) on $B_{2}$. Then $V$ is an admissible test function for the Rayleigh quotient of $\lambda_{T}\left(B_{1} \cup B_{2}\right)$, and

$$
\lambda_{T}\left(B_{1} \cup B_{2}\right) \leq \frac{\int_{B_{1} \cup B_{2}}|\nabla V|^{2} d x}{\int_{B_{1} \cup B_{2}} V^{2} d x}=\lambda_{\Delta}\left(B_{1} \cup B_{2}\right),
$$

and then $\lambda_{\Delta}\left(B_{1} \cup B_{2}\right)=\lambda_{T}\left(B_{1} \cup B_{2}\right)$.

\section{Proof of Theorem 1.1}

The proof of Theorem 1.1 will be pursued in two main steps. First, we show that the minimum of $\mu(\Omega, \alpha)$ among all sets of fixed measure is reached at the union of two disjoint balls. Second, we minimize among such sets.

3.1. An isoperimetric inequality for $\mu(\Omega, \alpha)$. The first step in order to prove Theorem 1.1 is to show an isoperimetric inequality for $\mu(\Omega, \alpha)$. To this aim, let

$$
\mathcal{B}(|\Omega|)=\left\{A=B_{1} \cup B_{2}: B_{1}, B_{2} \text { open disjoint balls of } \mathbb{R}^{n},\left|B_{1} \cup B_{2}\right|=|\Omega|\right\} .
$$

In the above definition we are implicitly assuming that $A \in \mathcal{B}(|\Omega|)$ can be a unique ball.

Proposition 3.1. Let $\Omega \subset \mathbb{R}^{n}$ be bounded, open set such that $\Omega \notin \mathcal{B}(|\Omega|)$. Then there exists $A_{\alpha}=B_{1} \cup B_{2} \in \mathcal{B}(|\Omega|)$ such that

$$
\mu(\Omega, \alpha)>\mu\left(A_{\alpha}, \alpha\right)=\min _{A \in \mathcal{B}(|\Omega|)} \mu(A, \alpha) .
$$

Moreover,

$$
\mu\left(A_{\alpha}, \alpha\right)=\mathcal{Q}\left(v_{1} \chi_{B_{1}}-v_{2} \chi_{B_{2}}, \alpha\right)
$$

for some nonnegative function $v_{1}$ and $v_{2}$, radially decreasing in $B_{1}$ and $B_{2}$ respectively. More precisely, either $v_{2} \equiv 0$, and $v_{1}$ is positive in $B_{1}=\Omega$, or $v_{1}>0$ in $B_{1}$ and $v_{2}>0$ in $B_{2}$. 
Proof. Let $u \in W_{0}^{1,2}(\Omega)$ be a minimizer of (1.1). Using (2.1) and Pólya-Szegö principle (2.2), we have that

$$
\begin{aligned}
\mu(\Omega, \alpha) & =\frac{\int_{\Omega_{+}}\left|\nabla u_{+}\right|^{2} d x+\int_{\Omega_{-}}\left|\nabla u_{-}\right|^{2} d x+\alpha\left|\int_{\Omega_{+}} u_{+}^{2} d x-\int_{\Omega_{-}} u_{-}^{2} d x\right|}{\int_{\Omega_{+}} u_{+}^{2} d x+\int_{\Omega_{-}} u_{-}^{2} d x} \\
& \geq \frac{\int_{\Omega_{+}^{\#}}\left|\nabla u_{+}^{\#}\right|^{2} d x+\int_{\Omega_{-}^{\#}}\left|\nabla u_{-}^{\#}\right|^{2} d x+\alpha\left|\int_{\Omega_{+}^{\#}}\left(u_{+}^{\#}\right)^{2} d x-\int_{\Omega_{-}^{\#}}\left(u_{-}^{\#}\right)^{2} d x\right|}{\int_{\Omega_{+}^{\#}}\left(u_{+}^{\#}\right)^{2} d x+\int_{\Omega_{-}^{\#}}\left(u_{-}^{\#}\right)^{2} d x} \\
& \geq \min _{\substack{w \in W_{0}^{1,2}\left(\Omega_{+}^{\#}\right) \\
z \in W_{0}^{1,2}\left(\Omega_{-}^{\#}\right)}} \frac{\int_{\Omega_{+}^{\#}}|\nabla w|^{2} d x+\int_{\Omega_{-}^{\#}}|\nabla z|^{2} d x+\alpha\left|\int_{\Omega_{+}^{\#}}\right| w\left|w d x+\int_{\Omega_{-}^{\#}}\right| z|z d x|}{\inf _{A \in \mathcal{B}(|\Omega|)} \mu(A ; \alpha) .}
\end{aligned}
$$

If $u_{+}$or $u_{-}$is not radially symmetric, then the inequality (3.2) is strict. Moreover, if $u_{+}$and $u_{-}$ are both radially decreasing functions, then $\Omega_{+}$and $\Omega_{-}$are balls such that $\left|\Omega_{+}\right|+\left|\Omega_{-}\right|<|\Omega|$, being $\Omega \notin \mathcal{B}(|\Omega|)$. The monotonicity of $\mu(\cdot ; \alpha)$ with respect to homotheties gives that in this case (3.3) is strict.

The arguments just used also give (3.1).

In order to conclude the proof of Theorem 1.1. we recall an isoperimetric inequality for $\lambda_{T}(\Omega)$ given in [17, which assures that if $B_{1}, B_{2}$ are disjoint balls with $\left|B_{1}\right|=\left|B_{2}\right|=|\Omega| / 2$, then

$$
\lambda_{T}(\Omega) \geq \lambda_{T}\left(B_{1} \cup B_{2}\right) .
$$

Proof of Theorem 1.1. If $\alpha \leq 0$, being $\mu(\Omega, \alpha)=\lambda_{\Delta}(\Omega)+\alpha$ the result is given by the wellknown Faber-Krahn inequality, which follows immediately from the Pólya-Szegö principle and the properties of rearrangements:

$$
\mathcal{Q}(u, \alpha) \geq \mathcal{Q}\left(u^{\#}, \alpha\right) \geq \mu\left(\Omega^{\#}, \alpha\right) .
$$

Then we can assume that $\alpha>0$.

Proposition 3.1 allows to restrict to the case $\Omega \in \mathcal{B}(|\Omega|)$. We denote by $\Omega_{d}$ the union of two disjoint balls with same measure, equal to $|\Omega| / 2$.

Then the proof is completed by observing that, by Proposition 2.1, and the Faber-Krahn inequality and (3.4), each eigencurve $\alpha \mapsto \mu(\Omega, \alpha), \alpha \geq 0$, is such that $\mu(\Omega, 0) \geq \mu\left(\Omega^{\#}, 0\right)=$ $\lambda_{\Delta}\left(\Omega^{\#}\right)$, then it increases linearly until it reaches the value $\lambda_{T}(\Omega)$ which is greater than $\lambda_{T}\left(\Omega_{d}\right)$ (see also Figure 1). More precisely, the eigencurve $\alpha \mapsto \mu(\Omega, \alpha)$ is above the curve

$$
\alpha \mapsto \begin{cases}\mu\left(\Omega^{\#}, \alpha\right) & \text { if } \alpha|\Omega|^{2 / n} \leq \alpha_{c}, \\ \mu\left(\Omega_{d}, \alpha\right) & \text { if } \alpha|\Omega|^{2 / n} \geq \alpha_{c},\end{cases}
$$

obtaining (1.2).

Acknowledgement. The author has been partially supported by Gruppo Nazionale per l'Analisi Matematica, la Probabilità e le loro Applicazioni (GNAMPA) of the Istituto Nazionale di Alta Matematica (INdAM), project "Diseguaglianze funzionali e problemi sovradeterminati" 2013. 


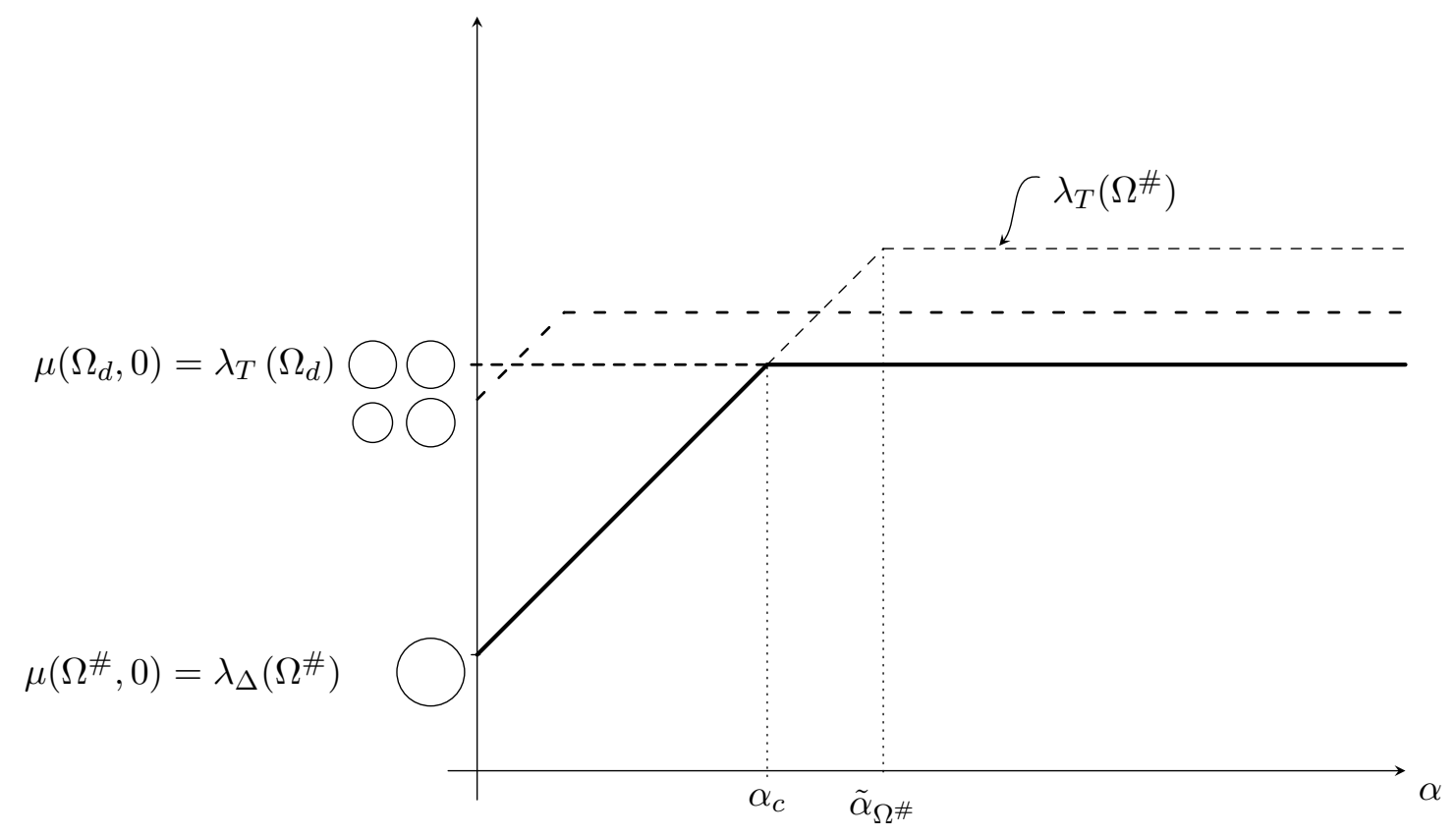

Figure 1 . A scheme of the eigencurves $\alpha \mapsto \lambda(\Omega, \alpha), \alpha \geq 0$, when $|\Omega|=\kappa$ is fixed. If $\Omega$ corresponds to $\Omega_{d}$ union of two disjoint balls of equal measure, then $\mu\left(\Omega_{d}, \alpha\right)$ is constant for $\alpha \geq 0$. Otherwise, $\mu(\Omega, \alpha)$ increases until it reaches its maximum value $\mu\left(\Omega, \alpha_{\Omega}\right)=\lambda_{T}(\Omega)$ in $\tilde{\alpha}_{\Omega}=\lambda_{T}(\Omega)-\lambda_{\Delta}(\Omega)$, then it is constant for $\alpha \geq \tilde{\alpha}_{\Omega}$. The solid line represents the values of $\min _{|\Omega|=\kappa} \mu(\Omega, \alpha)$.

\section{REFERENCES}

[1] B. Brandolini, F. Della Pietra, C. Nitsch and C. Trombetti, Symmetry breaking in a constrained Cheeger type isoperimetric inequality, ESAIM Control Optim. Calc. Var., to appear.

[2] B. Brandolini, P. Freitas, C. Nitsch and C. Trombetti, Sharp estimates and saturation phenomena for a nonlocal eigenvalue problem, Adv. Math., 228 (2011), 2352-2365.

[3] L. Brasco. On torsional rigidity and principal frequencies: an invitation to the Kohler-Jobin rearrangement technique, ESAIM Control Optim. Calc. Var., in press, DOI:10.1051/cocv/2013065.

[4] J. E. Brothers and W. P. . Ziemer, Minimal rearrangements of Sobolev functions, J. Reine Angew. Math., 384 (1988), 153-179.

[5] F. Chiacchio and G. di Blasio, Isoperimetric inequalities for the first Neumann eigenvalue in Gauss space, Annales de l'Institut Henri Poincaré (C) Non Linear Analysis, 29 (2012), 199-216.

[6] F. Della Pietra and N. Gavitone, Symmetrization for Neumann anisotropic problems and related questions, Advanced Nonlinear Stud., 12 (2012), 219-235.

[7] F. Della Pietra and N. Gavitone. Relative isoperimetric inequality in the plane: the anisotropic case. J. Convex. Anal., 20 (2013), 157-180.

[8] F. Della Pietra and N. Gavitone, Sharp bounds for the first eigenvalue and the torsional rigidity related to some anisotropic operators, Math. Nachr., 287 (2014), 194-209.

[9] F. Della Pietra and N. Gavitone, Stability results for some fully nonlinear eigenvalue estimates, Comm. Contemporary Math., in press, DOI:10.1142/S0219199713500399.

[10] F. Della Pietra and N. Gavitone, Upper bounds for the eigenvalues of Hessian equations, Annali Mat. Pura Appl., in press, DOI:10.1007/s10231-012-0307-5.

[11] F. Della Pietra and N. Gavitone, Faber-Krahn inequality for anisotropic eigenvalue problems with Robin boundary conditions, arXiv:1311.3456.

[12] L. Esposito, V. Ferone, B. Kawohl, C. Nitsch and C. Trombetti, The longest shortest fence and sharp Poincaré-Sobolev inequalities, Arch. Rational Mech. Anal., 206 (2012), 821-851.

[13] I.V. Gerasimov and A.I. Nazarov. Best constant in a three-parameter Poincaré inequality. Probl. Mat. Anal., 61 (2011), 69-86 (Russian). English transl.: J. Math. Sci., 179 (2011), 80-99. 
[14] A. Henrot, Extremum problems for eigenvalues of elliptic operators, Frontiers in Mathematics, Birkhauser, Basel, 2006.

[15] B. Kawohl, Rearrangements and convexity of level sets in P.D.E., Lecture notes in mathematics 1150 , Springer Verlag, Berlin, New York, 1985.

[16] V.G. Maz'ya. Sobolev spaces with applications to elliptic partial differential equations. Springer Verlag, Heidelberg, 2011.

[17] A.I. Nazarov. On symmetry and asymmetry in a problem of shape optimization. http://arxiv.org/abs/1208.3640 (2012), 1-5.

Francesco Della Pietra, Università degli studi di Napoli "Federico II", Dipartimento di Matematica e Applicazioni "R. Caccioppoli", Via Cintia, Complesso di Monte Sant'Angelo, I-80126 NAPOLI, ITALiA.

E-mail address: f.dellapietra@unina.it 